\title{
A Fuzzy Adaptive Firefly Algorithm for Multilevel Color Image Thresholding Based on Fuzzy Entropy
}

Yi Wang, Guangdong University of Science and Technology, Dongguan, China

Kangshun Li, South China Agricultural University, Guangzhou, China

\begin{abstract}
Multilevel thresholding image segmentation has been a hot issue of research in the last several years since it has a plenty of applications. The meta-heuristic search algorithm has unique advantages in solving multilevel threshold values. In this paper, a fuzzy adaptive firefly algorithm (FaFA) is proposed to solve the optimal multilevel thresholding for color images, and the fuzzy Kapur's entropy is considered as its objective function. In the FaFA, a fuzzy logical controller is designed to adjust the control parameters. A total of six satellite remote sensing color images are conducted in the experiments. The performance of the FaFA is compared with FA, BWO, SSA, NaFA, and ODFA. Some measure metrics are performed in the experiments. The experimental results show that the FaFA obviously outperforms other five algorithms.
\end{abstract}

\section{KEYWORDS}

Color Image Segmentation, Fuzzy Adaptive Firefly Algorithm, Fuzzy Kapur's Entropy, Multilevel Thresholding

\section{INTRODUCTION}

Image segmentation is an important method for image preprocessing, which has been widely applied to different engineering fields (Wang et al. 2018). For different image segmentation techniques, thresholding image segmentation is one of the popular techniques and has attracted a lot of attentions (Dhal et al. 2019). Generally, thresholding image segmentation is based on the gray histogram, and it is usually classified into two categories: bi-level and multilevel thresholds. if an image can be split into two regions, which is known as bi-level global thresholding. Assume that an image has multiple interesting parts, bi-level thresholding may not obtain the desirable results. However, multilevel thresholding should be taken into account to acquire the proper thresholds (Bhandari et al. 2018). Recently years, multilevel thresholding has attracted a lot of attentions, many researchers and scholars have put a plenty of effort into studying it. However, with the number of threshold value increases, the time complexity also increases exponentially since they search the optimal thresholding using exhaustive search method.

Satellite remote sensing color images have many applications including meteorological prediction, environmental protection and resource exploration. For color images, the images contain more

DOI: 10.4018/IJCINI.20211001.oa44

This article published as an Open Access article distributed under the terms of the Creative Commons Attribution License (http://creativecommons.org/licenses/by/4.0/) which permits unrestricted use, distribution, and production in any medium, provided the author of the original work and original publication source are properly credited. 
information and has three different color components, the time complexity will be exponential to the traditional exhaustive search method. Therefore, multilevel thresholding for color satellite image segmentation commonly be identified as a NP-hard optimization problem (Li and Wang. 2019). Facing multilevel thresholding problem, the computational complexity is an urgently problem to be solved. Traditional exhaustive search algorithm for multilevel thresholding are time expensive. Particularly, it will be a challenging task for multilevel thresholding image segmentation when dealing with remote sensing and satellite images (Jia et al. 2019). However, heuristic search algorithm has obvious advantages in searching the optimal value, so researchers have proposed plenty of optimization algorithms to solve multilevel thresholding problem, such as genetic algorithm (GA) (Manikandan et al. 2014), chaotic particle swarm optimization (CPSO) (Suresh et al. 2017), artificial bee colony algorithm (ABC) (Horng. 2011), modified bacterial foraging optimization (MBFO) (Tang et al. 2017) and ant colony optimization (ACO) (Khorram et al. 2019). Recently, a plenty of novel heuristic algorithms are reported, which are utilized to solve various optimization problems. Such as whale optimization algorithm (WOA) (Aziz et al. 2017), difference evolution (DE) (Li et al. 2019), improved bat algorithm (IBA) (Yun et al. 2019), Firefly algorithm (FA) (Pare et al. 2017), Salp Swarm Algorithm (SSA) (Wang et al. 2020), Electromagnetic field optimization (EFO) (Upadhyay et al. 2019), flower pollination algorithm (FPA) (Wang et al. 2015), black widow optimization (BWO) (Houssein et al. 2020), Harris hawks optimization (HHO) (Bao et al. 2019), moth-flame optimization (MFO) (Khairuzzaman et al. 2017), krill herd optimization (KHO) (Beevi et al .2016) and so on. Among them, the FA is a popular heuristic algorithm based on the firefly flight process. Unlike the ABC, GA and the above mentioned other algorithms. The FA has few control parameters, it is very easy to implement; and has been widely used for solving different types of optimization problems, including feature selection (Zhang et al. 2017), solar radiation prediction (Ibrahim et al. 2017), multilevel thresholding (Rajinikanth et al. 2015), many objective optimization and many others (Li and Chen. 2018).

Though the standard FA has been proved to be an effective optimization algorithm, it still should be enhanced when facing complex optimization problems. At present, a plenty of improvement FA have been reported, in which most of the variant FA focus on changing the control parameters. Raja N S et al. used a Levy flight to replaced the random search strategy in the standard FA. The experimental results indicated that the LFFA provide better optimization accuracy compared with FA (Raja et al. 2013). Fister et al. proposed 12 different chaotic maps to adjust the three parameters $\alpha$, $\beta$, and $\gamma$. The experimental results showed that chaotic map can effectively enhance the convergence rate and robustness of the standard FA (Fister et al. 2015). Yu et al. proposed a variable step size based firefly algorithm (VSSFA), in VSSFA, a dynamic decreasing function is utilized to model the change of parameter $\alpha$, the results showed that the proposed algorithm improved the performance of the standard FA (Yu et al. 2015). Baykasoglu A et al. proposed an adaptive firefly algorithm, the results showed the performance of the AFA was apparently improved when compared with FA (Baykasoglu et al. 2015). Verma O P et al. presented a opposition and dimensional based FA (ODFA), in ODFA, a opposition based methodology was proposed to initialize the population. The results indicated that the proposed algorithm achieved high convergence speed than FA, PSO, ACO, EFO and DE (Baykasoglu et al. 2015). H.Wang et al. regarded that the firefly would attract the nearest firefly, so they proposed a neighborhood attraction (NaFA). The experimental results showed that the NaFA was better than other six FA variants in term of time complexity and accuracy (Wang et al. 2017). Aref $Y$ et al. conceived a modified firefly algorithm (MFA), in which the tidal force formula was applied to enhance the FA, the study findings indicated the MFA outperforms other existing FA variants (Yelghi and Kose. 2018). All of the improvements were adopted to balance the exploitation and exploration capabilities of the algorithm. There is no single algorithm can solve every problem. When solving color image thresholding, The standard FA is still need to be improved.

In order to enhance the performance of the classic firefly algorithm and improve the efficiency of solving multilevel color image threshold value. In this paper, an adaptive firefly algorithm called FaFA is proposed to enhance the performance of the original FA. A fuzzy logic controller is used to 
adjust the three parameters. A total of six satellite images are utilized to estimate the performance of the proposed algorithm. Moreover, FA, SSA, BWO, ODFA and NaFA are introduced to compare with the FaFA. The corresponding measure indexes including the objective function value, PSNR, FSIM, $p$-value test and the performance of the convergence are adopted in the experiments.

The rest of the paper is organized as follows: The theory of fuzzy Kapur's entropy is explained in Section 2 . Section 3 introduces the firefly algorithm. The following, the framework of fuzzy adaptive firefly algorithm is shown in section 4 . Experiments results are analyzed in section 5. Conclusions and further work are drawn in section 6.

\section{THRESHOLDING IMAGE SEGMENTATION METHOD}

Multilevel thresholding for color image segmentation is to search for a series of suitable thresholds based on histograms of each color channel to split three components (red, green and blue) respectively. In generally, multilevel thresholding for color image segmentation based on heuristic algorithm often adopted some criterion such as Otsu, Tsallis entropy, Renyi entropy, cross entropy and fuzzy entropy (Pare et al. 2020). To obtain a better image segmentation results, we use a fuzzy Kapur's entropy to measure the segmentation image.

\subsection{Kapur's Entropy}

For color image, assume that each color component with $L$ gray levels and these gray levels are within the range of $[0, L-1]$. If the threshold vector $T=\left[t_{1}, t_{2}, \cdots, t_{n-1}\right]$ is made up of $n-1$ thresholds, which can segment an image into $n$ classes:

$$
\left\{\begin{array}{l}
I_{1}=\left\{(x, y) \mid 0 \leq g(x, y) \leq t_{1}-1\right\} \\
I_{2}=\left\{(x, y) \mid t_{1} \leq g(x, y) \leq t_{2}-1\right\} \\
\cdots \\
I_{n}=\left\{(x, y) \mid t_{n-1} \leq g(x, y) \leq L-1\right\}
\end{array}\right.
$$

Then, the probability $p_{i}$ of each gray level can be calculated by:

$$
p_{i}=\frac{f_{i}}{\sum_{i=0}^{L-1} f_{i}}, \quad \sum_{i=0}^{L-1} p_{i}=1
$$

where $f_{i}$ is the frequency of gray-level $i$.

Kapur's entropy method is to calculate the maximum value of the objective entropy function based on the histogram of the image, which can be easily extended to solve multilevel thresholds image segmentation problem: 


$$
\begin{aligned}
& H_{1}=-\sum_{i=0}^{t_{1}-1} \frac{p_{i}}{\omega_{1}} \ln \frac{p_{i}}{\omega_{1}}, \quad \omega_{1}=\sum_{i=0}^{t_{1}-1} p_{i} \\
& H_{2}=-\sum_{i=t_{1}}^{t_{2}-1} \frac{p_{i}}{\omega_{2}} \ln \frac{p_{i}}{\omega_{2}}, \quad \omega_{2}=\sum_{i=t_{1}}^{t_{2}-1} p_{i} \\
& H_{j}=-\sum_{i=t_{j-1}}^{t_{j}-1} \frac{p_{i}}{\omega_{j}} \ln \frac{p_{i}}{\omega_{j}}, \quad \omega_{j}=\sum_{i=t_{j-1}}^{t_{j}-1} p_{i} \\
& H_{n}=-\sum_{i=t_{n}}^{L-1} \frac{p_{i}}{\omega_{n}} \ln \frac{p_{i}}{\omega_{n}}, \quad \omega_{n}=\sum_{i=t_{n}}^{L-1} p_{i}
\end{aligned}
$$

where $H_{j}$ denotes the Kapur's entropy value of $j$-th part of segmented image. There are $n$ thresholds need to be solved, so it can be deemed as a $n$ dimensional optimization problem. The objective function is defined as:

$$
T^{*}=\arg \max \left(\sum_{i=0}^{m} H_{i}\right)
$$

\subsection{Fuzzy Kapur's Entropy Multilevel Thresholding}

In the fuzzy entropy theory, assume that the size of an image is $M_{*} N$, and let an image $I$ be $D=\{(i$, $j) \mid i=0, \ldots, M-1 ; j=0, \ldots, N-1\}$. If the image is divided into three regions named as $E_{d}, E_{m}, E_{b}$ by two thresholds. Usually, the histogram of the image can be computed by:

$$
h_{k}=\frac{n_{k}}{M * N}, \quad \sum_{k=0}^{255} h_{k}=1 .
$$

where $0 £ k £ 255 ; n_{k}$ is the number of occurrences of the $k$-th pixel in $D_{k} ; h_{k}$ is the probability of gray level $k$. Hence, the probability distribution of $E_{d}, E_{m}, E_{b}$ can be expressed as:

$$
p_{d}=P\left(E_{d}\right) ; \quad p_{m}=P\left(E_{m}\right) ; p_{b}=P\left(E_{b}\right)
$$

For each $(i, j) \in D$, let:

$$
\begin{aligned}
& D_{d}=\left\{(i, j) \mid 0 \leq g(i, j)<t_{1}\right\}, \\
& D_{m}=\left\{(i, j) \mid t_{1} \leq g(i, j)<t_{2}\right\}, \\
& D_{b}=\left\{(i, j) \mid t_{2} \leq g(i, j) \leq L-1\right\}
\end{aligned}
$$

Utilizing $\mu_{d}, \mu_{m}, \mu_{b}$ as the membership functions of $E_{d}, E_{m}, E_{b}$, which is shown in Figure 1 . There are six fuzzy parameters of $u_{1}, v_{1}, w_{1}, u_{2}, v_{2}, w_{2}$ in the membership functions.

Therefore, the thresholds $t_{1}$ and $t_{2}$ depend on the six fuzzy parameters. The probability distribution of the three regions can be calculated by the following formula (Naidu et al. 2017): 
Figure 1. Membership function graph

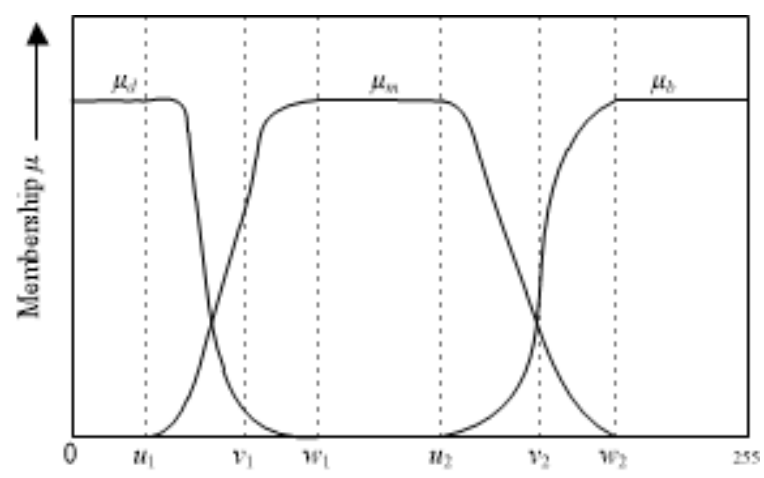

$$
\begin{aligned}
& p_{d}=\sum_{k=0}^{255} p_{k} \cdot p_{d \mid k}=\sum_{k=0}^{255} p_{k} \cdot \mu_{d}(k) \\
& p_{m}=\sum_{k=0}^{255} p_{k} \cdot p_{m \mid k}=\sum_{k=0}^{255} p_{k} \cdot \mu_{m}(k) \\
& p_{b}=\sum_{k=0}^{255} p_{k} \cdot p_{b \mid k}=\sum_{k=0}^{255} p_{k} \cdot \mu_{b}(k)
\end{aligned}
$$

where $p_{d l k}, p_{m l k},\left.p_{b}\right|_{k}$ represent the conditional probability and satisfy the constraint of $p_{d l k}+p_{m l k}+$ $\left.p_{b}\right|_{k}=1$. The corresponding mathematical formulas of the three membership functions are expressed as:

$$
\mu_{k}(k)=\left\{\begin{array}{lc}
1 & k \leq u_{1} \\
1-\frac{\left(k-u_{1}\right)^{2}}{\left(\omega_{1}-u_{1}\right) \cdot\left(v_{1}-u_{1}\right)} & u_{1} \leq k \leq v_{1} \\
\frac{\left(k-\omega_{1}\right)^{2}}{\left(\omega_{1}-u_{1}\right) \cdot\left(\omega_{1}-v_{1}\right)} & v_{1} \leq k \leq \omega_{1} \\
0 & k \geq \omega_{1}
\end{array}\right.
$$

$$
\mu_{b}(k)=\left\{\begin{array}{lr}
0 & k \leq u_{2} \\
\frac{\left(k-u_{2}\right)^{2}}{\left(\omega_{2}-u_{2}\right) \cdot\left(v_{2}-u_{2}\right)} & u_{2} \leq k \leq v_{2} \\
1-\frac{\left(k-\omega_{2}\right)^{2}}{\left(\omega_{2}-u_{2}\right) \cdot\left(\omega_{2}-v_{2}\right)} & v_{2} \leq k \leq \omega_{2} \\
1 & k \geq u_{2}
\end{array}\right.
$$




$$
\mu_{m}(k)=\left\{\begin{array}{lc}
0 & k \leq u_{1} \\
\frac{\left(k-u_{1}\right)^{2}}{\left(\omega_{1}-u_{1}\right) \cdot\left(v_{1}-u_{1}\right)} & u_{1} \leq k \leq v_{1} \\
1-\frac{\left(k-\omega_{1}\right)^{2}}{\left(\omega_{1}-u_{1}\right) \cdot\left(\omega_{1}-v_{1}\right)} & v_{1} \leq k \leq \omega_{1} \\
1 & \omega_{1} \leq k \leq u_{2}
\end{array}\right.
$$

where $u_{1}, v_{1}, w_{1}, u_{2}, v_{2}, w_{2}$ should satisfy the condition of $0 £ u_{1}<v_{1}<w_{1}<u_{2}<v_{2}<w_{2} £ 255$. Then, the fuzzy entropy of each part can be calculated by the following formulas:

$$
\begin{aligned}
& H_{d}=-\sum_{k=0}^{255} \frac{p_{k} \cdot \mu_{d}(k)}{p_{d}} \ln \left(\frac{p_{k} \cdot \mu_{d}(k)}{p_{d}}\right) \\
& H_{m}=-\sum_{k=0}^{255} \frac{p_{k} \cdot \mu_{m}(k)}{p_{m}} \ln \left(\frac{p_{k} \cdot \mu_{m}(k)}{p_{m}}\right) \\
& H_{b}=-\sum_{k=0}^{255} \frac{p_{k} \cdot \mu_{b}(k)}{p_{b}} \ln \left(\frac{p_{k} \cdot \mu_{b}(k)}{p_{b}}\right)
\end{aligned}
$$

The conventional fuzzy entropy function is to calculate the sum entropy of each part, and the optimal multilevel thresholding is to maximize the total fuzzy entropy (Song et al. 2019):

$$
H\left(u_{1}, v_{1}, w_{1}, u_{2}, v_{2}, w_{2}\right)=\arg \max \left(H_{d}+H_{m}+H_{b}\right)
$$

Hence, fuzzy entropy for bi-level thresholds color image segmentation need to solve six optimal parameters, the time consuming is expensive. However, the maximize fuzzy entropy does not to take into account the detail problem of an image.

As shown in Figure 1, the optimal membership value is the crossover points of membership function which can be expressed as:

$$
\begin{aligned}
& \mu_{d}\left(t_{1}\right)=\mu_{m}\left(t_{1}\right)=0.5 \\
& \mu_{m}\left(t_{2}\right)=\mu_{b}\left(t_{2}\right)=0.5
\end{aligned}
$$

According to the above equations, $t_{1}$ and $t_{2}$ can be calculated by the following formulas: 
$t_{1}=\left\{\begin{array}{lc}u_{1}+\sqrt{\left(w_{1}-u_{1}\right) \cdot\left(v_{1}-u_{1}\right) / 2} & \left(u_{1}+w_{1}\right) / 2<v_{1}<w_{1} \\ w_{1}-\sqrt{\left(w_{1}-u_{1}\right) \cdot\left(v_{1}-u_{1}\right) / 2} & u_{1}<v_{1}<\left(u_{1}+w_{1}\right) / 2\end{array}\right.$

$t_{2}= \begin{cases}u_{2}+\sqrt{\left(w_{2}-u_{2}\right) \cdot\left(v_{2}-u_{2}\right) / 2} & \left(u_{2}+w_{2}\right) / 2<v_{2}<w_{2} \\ w_{2}-\sqrt{\left(w_{2}-u_{2}\right) \cdot\left(v_{2}-u_{2}\right) / 2} & u_{2}<v_{2}<\left(u_{2}+w_{2}\right) / 2\end{cases}$

Fuzzy entropy thresholding also can extend to multilevel thresholds image segmentation. Due to one threshold is determined by three fuzzy parameters, so obtained $n$ thresholds, $3 n$ fuzzy parameters need to be solved. Hence, fuzzy entropy multilevel thresholding is time consuming.

\section{FIREFLY ALGORITHM}

The firefly algorithm simulates the behavior of the fireflies in nature searching for companions through their own light. Fireflies rely on the intensity of their own light to attract other companions. The stronger the light, the stronger the attraction. The light intensity $I(r)$ can be defined as (Yang, 2009):

$$
I(r)=I_{0} e^{-\gamma r^{2}}
$$

where $I_{0}$ represents the initial light intensity, $r$ is the distance between two fireflies, and $\gamma$ is a fixed light absorption coefficient. The attractiveness $\beta(r)$ based on the light intensity, that can be expressed as:

$$
\beta(r)=\beta_{0} e^{-\gamma r^{2}}
$$

where $\beta_{0}$ is the attractiveness at $r=0$.

The attractiveness $\beta$ depends on the distance $r$, the distance between any two fireflies $i$ and $j$ at $X_{i}$ and $X_{j}$ can be computed according to Euclidian distance:

$$
r_{i, j}=\left\|X_{i}-X_{j}\right\|=\sqrt{\sum_{k=1}^{d}\left(x_{i, k}-x_{j, k}\right)^{2}}
$$

where $x_{i, k}$ is the $k$-th element of the $i$-th firefly and $d$ is the dimension of the problem. Each firefly $i$ moves toward to firefly $j$, as follow:

$$
X_{i}=X_{i}+\beta_{0} e^{-\gamma r_{i, j}^{2}}\left(X_{j}-X_{i}\right)+\alpha\left(\operatorname{rand}-\frac{1}{2}\right)
$$

where, $\alpha$ is called step factor, rand represents uniformly distributed random number within [0, 1].The process of FA is summarized as the pseudo code shown in Table 1. 
Table 1. The pseudo code of firefly algorithm

1. Initialize $N$ fireflies $\left\{X_{1}(t), X_{2}(t), \ldots, X_{N}(t)\right\}$;

2. Evaluate the fitness values;

3. while $t £ G$

4. For $i=1: N$

5. For $j=1: N$

6. if $f\left(X_{j}(\mathrm{t})<f\left(X_{i}(\mathrm{t})\right)\right.$

7. Move firefly $i$ towards $j$ in all $d$ dimensions;

8. Calculate the fitness value of the new $X_{i}$;

9. end

10. end

11. end

12. $t++$;

13. end

14. Out the optimal solution.

\section{THE FUZZY ADAPTIVE FIREFLY ALGORITHM}

\subsection{Analysis the Parameters}

The performance of FA depends on its control parameters. Different parameters setting can lead completely different results. To tackle this problem, we will analyze the parameters of the FA, then some strategies are proposed to improve the FA. Now, let us assume that the FA convergences. Ideally, all the fireflies will cluster in the same position. Then, we can obtain the following equations:

$\lim _{t \rightarrow \infty} X_{i}(t)=\lim _{t \rightarrow \infty} X_{j}(t)=\lim _{t \rightarrow \infty} X_{i}(t+1), \quad \forall i \neq j$

Therefore, the following conclusions can be drawn:

$$
\lim _{t \rightarrow \infty}\left(X_{i}(t+1)-X_{i}(t)\right)=0
$$

$\lim _{t \rightarrow \infty}\left(X_{i}(t)-X_{j}(t)\right)=0$

According to Equation (22):

$$
X_{i}(t+1)-X_{i}(t)=\beta_{0} e^{-\gamma r_{i, j}^{2}}\left(X_{j}(t)-X_{i}(t)\right)+\alpha\left(\operatorname{rand}-\frac{1}{2}\right)
$$

Then

$$
\lim _{t \rightarrow \infty}\left(X_{i}(t+1)-X_{i}(t)\right)=\lim _{t \rightarrow \infty}\left(\beta_{0} e^{-\gamma r_{i, j}^{2}}\left(X_{j}(t)-X_{i}(t)\right)+\alpha\left(\operatorname{rand}-\frac{1}{2}\right)\right)
$$


So, it is easy to get the following inferences:

$$
\begin{aligned}
& \lim _{t \rightarrow \infty}\left(\beta_{0} e^{-\gamma r_{i, j}^{2}}\left(X_{j}(t)-X_{i}(t)\right)+\alpha\left(\text { rand }-\frac{1}{2}\right)\right)=0 \\
& \Rightarrow \lim _{t \rightarrow \infty}\left(\beta_{0} e^{-\gamma r_{i, j}^{2}}\right) \cdot \lim _{t \rightarrow \infty}\left(X_{j}(t)-X_{i}(t)\right)+\lim _{t \rightarrow \infty} \alpha\left(\text { rand }-\frac{1}{2}\right)=0 \\
& \Rightarrow\left(\operatorname{rand}-\frac{1}{2}\right) \lim _{t \rightarrow \infty} \alpha=0 \\
& \Rightarrow \lim _{t \rightarrow \infty} \alpha=0
\end{aligned}
$$

Hence, we can summarize that the parameter $\alpha$ should approach to zero at the end of generations. Generally, $\alpha \in[0,1]$.

According to the formulas (20) and (21), the attractiveness $\beta(r)$ is determined by the distance among two fireflies. However, the distance will gradually decrease during the evolutionary process, then if the algorithm convergences, the distance will turn into zero, so we can gain:

$$
\lim _{t \rightarrow \infty} r_{i, j}=0
$$

then

$$
\lim _{t \rightarrow \infty} \beta(r)=\lim _{t \rightarrow \infty} \beta_{0} e^{-\gamma r^{2}}=\beta_{0} \lim _{t \rightarrow \infty} e^{0}=\beta_{0}
$$

From the above analysis, the attractiveness $\beta(r)$ will gradually close to $\beta_{0}$ during the later phase. Generally, $\beta_{0}=1$. In the following, we will discuss the parameter $\gamma$. If $\gamma{ }^{\circledR} \infty$, then, the following formula can be obtained:

$$
\lim _{\gamma \rightarrow \infty} \beta_{0} e^{-\gamma r_{i, j}^{2}}=\lim _{\gamma \rightarrow \infty} \beta_{0} e^{-\infty}=0
$$

As a results, the Equation (22) can turn into the following formula:

$$
X_{i}=X_{i}+\alpha\left(\operatorname{rand}-\frac{1}{2}\right)
$$

This means the FA just can use random steps for search the space problem, it can be considered as a local search. And also, if $\gamma{ }^{\circledR 0}$.

$$
\lim _{\gamma \rightarrow 0} \beta_{0} e^{-\gamma r_{i, j}^{2}}=\lim _{\gamma \rightarrow 0} \beta_{0} e^{0}=\beta_{0}
$$

Then, the Equation (22) can turn into the following expression: 
Figure 2. The curve of three parameters with growth of generations

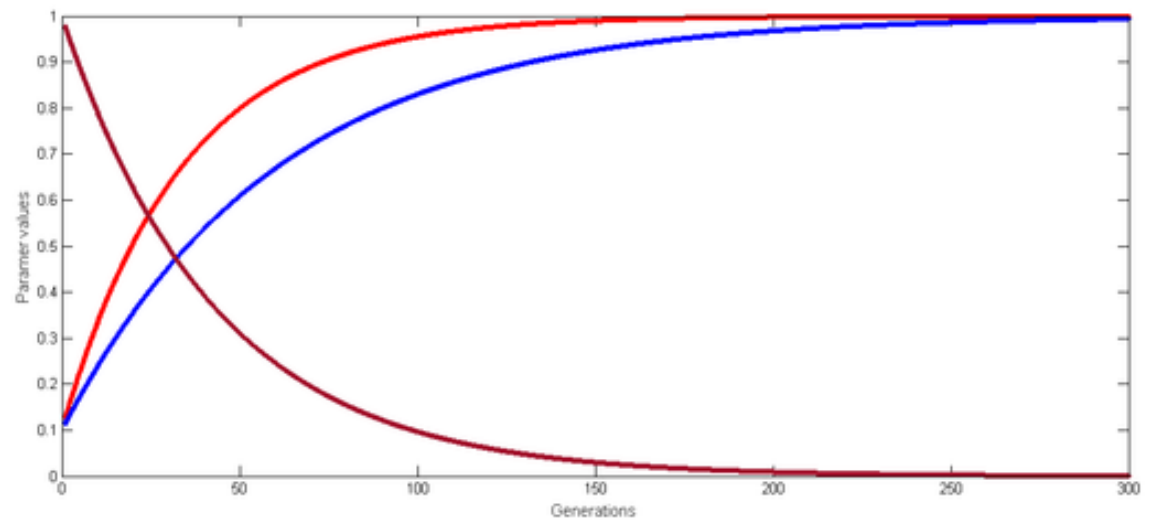

$$
X_{i}=X_{i}+\beta_{0}\left(X_{j}-X_{i}\right)+\alpha\left(\operatorname{rand}-\frac{1}{2}\right)
$$

This Means that all the fireflies in the population are basically together. In this situation, it can be regarded as a global search. Hence, they are two movements in the FA. These two movements indicate local and global search in firefly algorithm. So, keeping the balance between local and global search during the evolution process, which is determined by the parameter $\gamma$. At the beginning of iterations, the setting of $\gamma$ should be small, it is helpful to find the globally optimal solution. On the contrary, the setting of $\gamma$ should be large, it is helpful to find the locally optimal solution.

In theory, $\gamma \in[0, \infty)$. In fact, its setting depends on the optimization problem, it typically varies from 0.1 to 10. After the above analysis, when $\beta(t) \in[0,1], \gamma(t) \in[0,1], \alpha(t) \in[0,1]$, the curve of the the three parameters are given in Figure 2.

\subsection{The Design of Fuzzy Logic Controller}

After the above analysis, and from the Figure 2, we can draw the following conclusions:

(1) when the maximum objective function value is found at the end of the generation, low the step factor $\alpha$ and high light absorption coefficient $\gamma$ and attractiveness $\beta$ are often preferred.

(2) when the local optimal value kept at one value for a long time, and it's close to satisfying the termination condition. The light absorption coefficient $\gamma$ and attractiveness $\beta$ should decrease, the step factor $\alpha$ should increase.

Therefore, a fuzzy system is designed to tune the three control parameters. The best fitness value $(B F V)$ and the number of generations $(G)$ as the input variables, and the step factor $\alpha$ and light absorption coefficient $\gamma$ and attractiveness $\beta$ as the output variables. Different optimization problems have different ranges of $B F V$ and $G$. So, we normalize the parameters. The $B F V$ can be normalized using the following formula: 
Figure 3. Membership functions of inputs and outputs (a) NBF or NU, (b) $\alpha$, (c) $\beta$, (d) $Y$

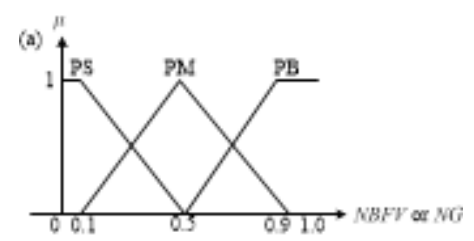

Table 2. Fuzzy rules for step factor $a$

\begin{tabular}{|l|l|l|l|l|}
\hline \multicolumn{2}{|c|}{$\alpha$} & \multicolumn{3}{c|}{$N G$} \\
\cline { 3 - 5 } \multicolumn{2}{|c|}{ PS } & \multicolumn{2}{c|}{ PM } & PB \\
\hline \multirow{3}{*}{$N B F$} & PS & PS & PM & PS \\
& PM & PM & PM & PS \\
& PB & PM & PS \\
\hline
\end{tabular}

$N B F V=\frac{B F V-B F V_{\min }}{B F V_{\max }-B F V_{\min }}$

where, $B F V_{\max }$ and $B F V_{\text {min }}$ is the maximum and minimum values of $B F V$, respectively. The $G$ can be normalized as:

$N G=\frac{G-G_{\max }}{G_{\max }-G_{\min }}$

where, $G_{\max }$ and $G_{\min }$ is the maximum and minimum values of $G$, respectively. The bound values for the parameters $\alpha(t) \in[0,1], \beta(t) \in[0,1], \gamma(t) \in[0.1,10]$.

The membership functions of every input and output are shown in Figure 3 . The fuzzy rules of the step factor $(\alpha)$, the attractiveness $(\beta)$, the light absorption coefficient $(\gamma)$ are shown in Tables 2-4. According to Tables 2-4, PS (positive small), PM (positive medium) and PB (positive big) are the linguistic values for all of the input and output variables.

According to the above description, the framework of our proposed FaFA is presented in Table 5.

Table 3. Fuzzy rules for the attractiveness $\beta$

\begin{tabular}{|l|l|l|l|l|}
\hline \multicolumn{2}{|c|}{$\beta$} & \multicolumn{3}{c|}{$N G$} \\
\cline { 3 - 5 } \multicolumn{2}{|c|}{$\beta$} & \multicolumn{2}{c|}{ PS } & \multicolumn{2}{c|}{ PM } \\
\hline \multirow{3}{*}{$N B F$} & PS & PS & PM & PM \\
& PM & PM & PM & PM \\
& PB & PM & PM & \\
\hline
\end{tabular}


Table 4. Fuzzy rules for the light absorption coefficient $y$

\begin{tabular}{|c|c|c|c|c|}
\hline & & & $N G$ & \\
\hline & & PS & PM & PB \\
\hline$N B F$ & $\begin{array}{l}\mathrm{PS} \\
\mathrm{PM} \\
\mathrm{PB}\end{array}$ & $\begin{array}{l}\text { PS } \\
\text { PM } \\
\text { PM }\end{array}$ & \begin{tabular}{|l} 
PM \\
PM \\
PB
\end{tabular} & $\begin{array}{l}\text { PB } \\
\text { PB } \\
\text { PB }\end{array}$ \\
\hline
\end{tabular}

\section{EXPERIMENTS AND ANALYSIS}

In this section, the test color images and the corresponding histograms are presented firstly, then the parameters setting of five heuristic algorithms and the experimental conditions are listed. Finally, a set of evaluation indicators including objective function value, PSNR, FSIM, convergence performance and Wilcoxon rank sum test are utilized to evaluate the performance of the proposed algorithm.

\subsection{Experimental Setting}

To verify the superiority of the proposed algorithm, other five evolutionary algorithms, namely, NaFA, BWO, ODFA, SSA, and FA are selected to compare with the FaFA, these algorithms were utilized in previous works and proved perfect performance. Particularly, NaFA, ODFA are the FA variants, BWO and SSA are recently proposed. The parameters setting of these algorithms are derived from the corresponding references. The maximum number of iterations of all the algorithms used in the experiment is set to 300 with a total of 30 times independently runs per algorithm for each test image, and the population size also sets to 30 . Each test image carries out in the experiments with the following number of thresholds: 5, 8, 10 and 12 .

\subsection{Test Images}

The test satellite color images used in the experiment are labeled 'Image 1', 'Image 2', 'Image 3', 'Image 4', 'Image 5' and 'Image 6'. The sizes of all images are 512×512. These original test images and their histograms are shown in Figure 4.

Table 5. Framework of the proposed FaFA

The Fuzzy Adaptive Firefly Algorithm

1. Initialize $N$ fireflies $\left\{X_{1}, X_{2}, \ldots, X_{N}\right\}$;

2. Evaluate the fitness values;

3. while $G £ G_{\max }$

4. Update the parameters according the fuzzy controller;

5. For $i=1: N$

6. For $j=1: N$

7. if $f\left(\left(X_{j}\right)<f\left(X_{i}\right)\right.$

8. Move firefly $i$ towards $j$ in all $d$ dimensions;

9. Calculate the fitness value of the new $X_{i}$;

10. end

11. end

12. end

13. $G++$;

14. end

15. Out the optimal solution. 


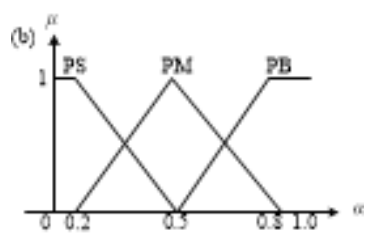

\subsection{Results on the Objective Function Value}

The proposed algorithm is utilized to solve the optimal multilevel thresholding for color images which regards the fuzzy Kapur's entropy as its objective function. It means the larger the objective function value, the better. Other five algorithms are introduced to compared with the FaFA. Then, comparing the performance of the algorithms. In detail, the mean objective function values and the corresponding standard deviation values are shown in Table 6, where ' $T$ ' ' is the number of the thresholds. The largest objective function value in each case are shown in bold.

In Table 6 the FaFA obtains the best results in 27 cases (30 in total) which shows the best performance in algorithm accuracy, nevertheless other five algorithms do not show superiority in any case when compared with the FaFA, the NaFA get the second rank followed by FaFA, and the BWO and ODFA perform nearly equally, and the performance of FA is the worst. The standard deviation of objective function values, also support the conclusion is that the proposed algorithm is much better than the other five algorithms.

\subsection{Segmentation Image Quality Metrics}

To access the performance of the proposed algorithm for color image segmentation, and avoiding the randomness in the results, Two image quality measurement indicators including Peak Signal-to-Noise Ration (PSNR) and Feature Similarity Index (FSIM) are utilized to estimate the segmented image quality. The PSNR metric is defined as (Khairuzzaman et al. 2017):

$$
P S N R=10 \log _{10}\left(\frac{255^{2}}{M S E}\right), \quad(d B)
$$

where, MSE is the mean square error.

$$
M S E=\frac{1}{M \cdot N} \sum_{i=1}^{M} \sum_{j=1}^{N}(I(i, j)-C(i, j))^{2}
$$

where, $C$ represents the original image and $I$ stands for the segmented image, $M \cdot N$ is the size of the image. The larger the PSNR value, the better the image segmentation effect.

The FSIM is adopted to evaluate the feature similarity between two images (Elaziz et al. 2019). It is calculated by:

$$
F S I M=\frac{\sum_{x \in \Omega} S_{L}(x) P C_{m}(x)}{\sum_{x \in \Omega} P C_{m}(x)}
$$


Table 6. Comparison of the average objective function values and standard deviation

\begin{tabular}{|c|c|c|c|c|c|c|c|}
\hline \multirow[t]{2}{*}{ Images } & \multirow[t]{2}{*}{$T$} & \multicolumn{6}{|c|}{ Average Objective Function Values and Standard Deviation } \\
\hline & & $\mathrm{NaFA}$ & SSA & $O D F A$ & $F A$ & $B W O$ & FaFA \\
\hline \multirow[t]{4}{*}{ Image 1} & 5 & $21.5762(5.69 \mathrm{E}-06)$ & $21.4328(6.89 \mathrm{E}-06)$ & $21.5016(9.86 \mathrm{E}-07)$ & $21.3254(9.46 \mathrm{E}-06)$ & $21.4896(5.97 \mathrm{E}-06)$ & $21.6134(1.26 E-07)$ \\
\hline & 8 & $29.2130(7.86 \mathrm{E}-05)$ & $28.6348(8.12 \mathrm{E}-04)$ & $29.0899(6.48 \mathrm{E}-05)$ & $28.4698(5.27 \mathrm{E}-04)$ & 29.1538(3.54E-05) & $29.2864(3.14 E-05)$ \\
\hline & 10 & $33.5256(8.56 \mathrm{E}-04)$ & $33.4542(1.65 \mathrm{E}-03)$ & $3.2263(9.82 \mathrm{E}-04)$ & $33.1256(6.38 \mathrm{E}-02)$ & $33.4527(7.98 \mathrm{E}-04)$ & $33.6548(6.25 E-04)$ \\
\hline & 12 & $37.7184(5.26 \mathrm{E}-03)$ & $37.1981(2.98 \mathrm{E}-02)$ & $37.3238(2.68 \mathrm{E}-02)$ & $37.1253(9.22 \mathrm{E}-02)$ & 37.5891(8.62E-03) & $37.7221(2.18 E-03)$ \\
\hline \multirow[t]{4}{*}{ Image 2} & 5 & $22.4570(1.14 E-08)$ & $22.3423(6.85 \mathrm{E}-07)$ & $22.4046(7.36 \mathrm{E}-09)$ & $22.3122(8.97 \mathrm{E}-07)$ & $22.3864(8.93 \mathrm{E}-09)$ & $22.4353(5.23 \mathrm{E}-09)$ \\
\hline & 8 & $29.8583(8.29 \mathrm{E}-06)$ & $29.7016(4.56 \mathrm{E}-05)$ & $29.8014(4.63 \mathrm{E}-07)$ & $29.5893(7.98 \mathrm{E}-05)$ & $29.7865(6.28 \mathrm{E}-06)$ & $30.0236(4.18 E-07)$ \\
\hline & 10 & $34.3364(5.96 \mathrm{E}-05)$ & $34.2238(7.39 \mathrm{E}-04)$ & $34.1912(7.21 \mathrm{E}-05)$ & $34.1365(4.33 \mathrm{E}-03)$ & $34.3012(7.37 \mathrm{E}-05)$ & $34.5292(3.86 E-06)$ \\
\hline & 12 & $38.4682(5.57 \mathrm{E}-03)$ & $38.2287(2.01 \mathrm{E}-02)$ & $38.2880(3.22 \mathrm{E}-03)$ & $38.2056(6.67 \mathrm{E}-02)$ & $38.3451(8.86 \mathrm{E}-03)$ & $38.6469(1.94 \mathrm{E}-05)$ \\
\hline \multirow[t]{4}{*}{ Image 3} & 5 & $20.5713(6.67 \mathrm{E}-07)$ & $20.2212(3.99 \mathrm{E}-06)$ & $20.5124(8.96 \mathrm{E}-07)$ & $20.1354(5.91 \mathrm{E}-06)$ & $20.4326(5.77 \mathrm{E}-07)$ & $20.6187(9.87 E-08)$ \\
\hline & 8 & $27.6569(5.41 \mathrm{E}-06)$ & $27.2401(4.48 \mathrm{E}-04)$ & $27.4059(4.67 \mathrm{E}-05)$ & $27.1365(3.35 \mathrm{E}-04)$ & $27.7634(6.89 \mathrm{E}-06)$ & $27.7380(6.59 \mathrm{E}-07)$ \\
\hline & 10 & $32.3372(4.33 \mathrm{E}-05)$ & $31.6709(8.29 \mathrm{E}-04)$ & $32.1320(3.37 \mathrm{E}-04)$ & $31.5569(9.01 \mathrm{E}-02)$ & $32.2869(7.61 \mathrm{E}-05)$ & $32.3376(3.68 \mathrm{E}-05)$ \\
\hline & 12 & $36.5716(8.62 \mathrm{E}-04)$ & $36.4026(7.02 \mathrm{E}-03)$ & $36.4086(1.64 \mathrm{E}-04)$ & $36.0347(4.57 \mathrm{E}-01)$ & $36.4102(8.13 \mathrm{E}-04)$ & $36.7373(9.54 \mathrm{E}-05)$ \\
\hline \multirow[t]{4}{*}{ Image 4} & 5 & $21.4959(9.29 \mathrm{E}-07)$ & $21.3901(3.96 \mathrm{E}-06)$ & 21.6143(4.31E-07) & $21.2356(8.69 \mathrm{E}-07)$ & $21.3693(6.99 \mathrm{E}-07)$ & $21.5841(5.72 \mathrm{E}-07)$ \\
\hline & 8 & $29.2448(7.61 \mathrm{E}-05)$ & $28.6814(5.92 \mathrm{E}-04)$ & $29.1488(1.57 \mathrm{E}-04)$ & $28.5642(7.20 \mathrm{E}-05)$ & $29.1245(6.78 \mathrm{E}-06)$ & $29.3541(2.39 \mathrm{E}-06)$ \\
\hline & 10 & $33.7535(9.25 \mathrm{E}-05)$ & $33.0666(8.63 \mathrm{E}-03)$ & $33.5273(6.33 \mathrm{E}-04)$ & $33.0354(5.63 \mathrm{E}-04)$ & $33.6524(8.96 \mathrm{E}-05)$ & $34.1201(4.59 E-05)$ \\
\hline & 12 & $37.7927(4.62 \mathrm{E}-03)$ & $36.8502(6.31 \mathrm{E}-02)$ & $37.1648(8.97 \mathrm{E}-03)$ & $37.2659(2.28 \mathrm{E}-02)$ & $36.7952(1.24 \mathrm{E}-05)$ & $37.9542(5.91 E-04)$ \\
\hline \multirow[t]{4}{*}{ Image 5} & 5 & $21.3851(8.86 \mathrm{E}-08)$ & $21.1634(8.65 \mathrm{E}-07)$ & 21.1943(6.37E-09) & $21.1024(8.10 \mathrm{E}-07)$ & $21.2302(3.26 \mathrm{E}-09)$ & $21.4365(5.36 \mathrm{E}-10)$ \\
\hline & 8 & $28.5824(4.59 \mathrm{E}-07)$ & $28.5578(9.86 \mathrm{E}-06)$ & $28.4251(8.95 \mathrm{E}-07)$ & $28.3695(6.07 \mathrm{E}-05)$ & $28.4867(5.43 \mathrm{E}-07)$ & $28.6953(7.78 E-08)$ \\
\hline & 10 & $32.8165(7.11 \mathrm{E}-07)$ & $32.5090(4.67 \mathrm{E}-04)$ & $32.6598(6.12 \mathrm{E}-06)$ & $32.4967(4.99 \mathrm{E}-03)$ & 32.7243(9.77E-06) & $32.9846(2.33 E-07)$ \\
\hline & 12 & $36.7466(3.67 \mathrm{E}-04)$ & 36.3301(3.94E-03) & $36.5673(7.73 \mathrm{E}-04)$ & $36.2861(2.27 \mathrm{E}-02)$ & $36.6645(1.67 \mathrm{E}-05)$ & $36.8697(8.54 \mathrm{E}-05)$ \\
\hline \multirow[t]{4}{*}{ Image 6} & 5 & $0.9151(2.28 \mathrm{E}-07)$ & $20.6474(1.29 \mathrm{E}-07)$ & $20.7599(8.66 \mathrm{E}-07)$ & $20.5634(5.38 \mathrm{E}-07)$ & 20.8651(3.95E-07) & $21.2138(6.29 \mathrm{E}-08)$ \\
\hline & 8 & $28.0419(8.63 \mathrm{E}-06)$ & $27.3719(6.48 \mathrm{E}-05)$ & $27.8685(5.97 \mathrm{E}-06)$ & $27.2563(4.91 \mathrm{E}-05)$ & $27.8967(5.46 \mathrm{E}-06)$ & $28.2657(9.11 E-07)$ \\
\hline & 10 & $32.5295(3.23 \mathrm{E}-05)$ & 31.1992(1.12E-04) & $32.2689(8.02 \mathrm{E}-05)$ & $31.1161(3.90 \mathrm{E}-04)$ & $32.4681(6.85 \mathrm{E}-05)$ & $32.6753(5.57 \mathrm{E}-06)$ \\
\hline & 12 & $36.4846(6.11 \mathrm{E}-04)$ & $35.8871(8.37 \mathrm{E}-03)$ & $36.1254(9.20 \mathrm{E}-04)$ & $35.4652(2.04 \mathrm{E}-02)$ & $36.3956(9.69 \mathrm{E}-04)$ & $36.5124(4.39 \mathrm{E}-04)$ \\
\hline
\end{tabular}

where

$S_{L}(x)=S_{P C}(x) S_{G}(x)$

$S_{P C}(x)=\frac{2 P C_{1}(x) P C_{2}(x)+T_{1}}{P C_{1}^{2}(x)+P C_{2}^{2}(x)+T_{1}} ;$

$S_{G}(x)=\frac{2 G_{1}(x) G_{2}(x)+T_{2}}{G_{1}^{2}(x)+G_{2}{ }^{2}(x)+T_{2}} ;$

$P C_{m}(x)=\max \left\{P C_{1}(x), P C_{2}(x)\right\}$.

where $T_{1}$ and $T_{2}$ are constants. $G(x)$ is the gradient magnitude of an image, and $P C(x)$ represents the phase congruence of an image. Here, we choose $T_{1}=0.85$ and $T_{2}=160$ in the experiments. A higher value of FSIM implies better performance.

The PSNR and FSIM results of the six test images are calculated by the involved algorithms, which are listed in Table 7 and Table 8 . It can be easily to find that the FaFA wins 27 times in total 30 comparisons on PSNR, and Table 8 shows that the FaFA wins 28 times in total 30 comparisons on FSIM. All the experimental results show that the proposed FaFA is the best algorithm in comparison with the quality of the segmented images.

\subsection{Convergence Performance}

To further access the FaFA, the convergence performance on 12 levels of thresholding is also studied. For the sake of fairness, All the parameters and settings keep it the same as the previous setting. In the same way, the six test images are tested to exemplify the performance. The results are shown in Figure 5. it can be found in Figure 5 that most of the algorithms like SSA, ODFA, BWO, NaFA and 
Table 7. Comparison of the average PSNR

\begin{tabular}{|c|c|c|c|c|c|c|c|}
\hline \multirow[t]{2}{*}{ Test Images } & \multirow[t]{2}{*}{$T$} & \multicolumn{6}{|c|}{ The Average PSNR } \\
\hline & & $N a F A$ & $S S A$ & $O D F A$ & $F A$ & $B W O$ & FaFA \\
\hline \multirow[t]{4}{*}{ Image 1} & 5 & 20.6676 & 20.4376 & 20.4567 & 20.1123 & 20.5568 & 21.2641 \\
\hline & 8 & 24.3047 & 23.4907 & 23.7378 & 23.0659 & 24.1236 & 24.3827 \\
\hline & 10 & 25.1320 & 24.1958 & 25.0864 & 23.7536 & 25.0124 & 25.2841 \\
\hline & 12 & 26.5284 & 25.9120 & 26.4550 & 25.1568 & 26.4851 & 26.6124 \\
\hline \multirow[t]{4}{*}{ Image 2} & 5 & 20.2866 & 18.9355 & 20.2763 & 19.2954 & 20.1986 & 20.3012 \\
\hline & 8 & 23.5917 & 23.3285 & 23.0385 & 22.0245 & 23.4893 & 23.5890 \\
\hline & 10 & 25.2743 & 24.8816 & 24.7235 & 24.1369 & 25.1698 & 25.8642 \\
\hline & 12 & 26.8006 & 24.1615 & 26.5675 & 25.8032 & 26.7851 & 26.9142 \\
\hline \multirow[t]{4}{*}{ Image 3} & 5 & 20.5375 & 19.4044 & 20.4606 & 19.1564 & 20.4865 & 20.6012 \\
\hline & 8 & 22.3450 & 20.9912 & 22.3012 & 21.3134 & 22.2639 & 22.4325 \\
\hline & 10 & 24.6083 & 24.1121 & 23.8006 & 22.5124 & 24.5123 & 23.7273 \\
\hline & 12 & 26.2609 & 26.1128 & 26.1728 & 25.9213 & 26.1352 & 26.3124 \\
\hline \multirow[t]{4}{*}{ Image 4} & 5 & 19.5021 & 18.7714 & 19.1242 & 18.3879 & 19.4568 & 19.6897 \\
\hline & 8 & 24.1292 & 21.7885 & 23.0387 & 22.6854 & 24.0986 & 24.3589 \\
\hline & 10 & 25.5346 & 23.0229 & 24.1923 & 22.7576 & 25.3652 & 25.6354 \\
\hline & 12 & 26.9231 & 26.2084 & 26.6812 & 25.8365 & 26.7956 & 27.1243 \\
\hline \multirow[t]{4}{*}{ Image 5} & 5 & 19.9503 & 19.8559 & 19.9124 & 19.0865 & 19.8567 & 20.1234 \\
\hline & 8 & 23.1050 & 22.4145 & 22.8451 & 21.3142 & 22.8659 & 23.4265 \\
\hline & 10 & 25.5621 & 23.8770 & 24.6983 & 23.1983 & 25.3659 & 25.7698 \\
\hline & 12 & 27.0504 & 25.5482 & 26.8457 & 26.0546 & 26.8956 & 27.2568 \\
\hline \multirow[t]{4}{*}{ Image 6} & 5 & 19.9213 & 18.8301 & 19.4521 & 18.7356 & 19.8653 & 19.9854 \\
\hline & 8 & 22.6605 & 21.2094 & 21.8647 & 21.1568 & 22.8693 & 22.8564 \\
\hline & 10 & 25.0969 & 23.1463 & 24.6983 & 23.0012 & 24.8698 & 25.2689 \\
\hline & 12 & 26.2698 & 25.3228 & 25.9752 & 25.2598 & 26.0243 & 26.3546 \\
\hline
\end{tabular}

Table 8. Comparison of the average FSIM

\begin{tabular}{|c|c|c|c|c|c|c|c|}
\hline \multirow[t]{2}{*}{ Test Images } & \multirow[t]{2}{*}{$T$} & \multicolumn{6}{|c|}{ The Average FSIM } \\
\hline & & $N a F A$ & $S S A$ & $O D F A$ & $F A$ & $B W O$ & $F a A$ \\
\hline \multirow{4}{*}{ Image 1} & 5 & 0.8813 & 0.8782 & 0.8795 & 0.8698 & 0.8769 & 0.8898 \\
\hline & 8 & 0.9343 & 0.9075 & 0.9275 & 0.9021 & 0.9256 & 0.9366 \\
\hline & 10 & 0.9507 & 0.9378 & 0.9486 & 0.9265 & 0.9457 & 0.9532 \\
\hline & 12 & 0.9669 & 0.9453 & 0.9658 & 0.9356 & 0.9586 & 0.9670 \\
\hline \multirow[t]{4}{*}{ Image 2} & 5 & 0.9413 & 0.9314 & 0.9403 & 0.9286 & 0.9365 & 0.9522 \\
\hline & 8 & 0.9713 & 0.9694 & 0.9705 & 0.9536 & 0.9772 & 0.9764 \\
\hline & 10 & 0.9792 & 0.9761 & 0.9768 & 0.9689 & 0.9752 & 0.9861 \\
\hline & 12 & 0.9853 & 0.9821 & 0.9831 & 0.9810 & 0.9810 & 0.9892 \\
\hline \multirow[t]{4}{*}{ Image 3} & 5 & 0.8668 & 0.8404 & 0.8654 & 0.8354 & 0.8569 & 0.8673 \\
\hline & 8 & 0.9027 & 0.9072 & 0.9012 & 0.8968 & 0.9012 & 0.9095 \\
\hline & 10 & 0.9315 & 0.9257 & 0.9260 & 0.9186 & 0.9286 & 0.9412 \\
\hline & 12 & 0.9620 & 0.9509 & 0.9502 & 0.9486 & 0.9586 & 0.9645 \\
\hline \multirow{4}{*}{ Image 4} & 5 & 0.9155 & 0.9021 & 0.9286 & 0.9015 & 0.9102 & 0.9315 \\
\hline & 8 & 0.9792 & 0.9664 & 0.9681 & 0.9568 & 0.9685 & 0.9786 \\
\hline & 10 & 0.9810 & 0.9546 & 0.9692 & 0.9489 & 0.9789 & 0.9854 \\
\hline & 12 & 0.9869 & 0.9732 & 0.9797 & 0.9625 & 0.9812 & 0.9880 \\
\hline \multirow[t]{4}{*}{ Image 5} & 5 & 0.9394 & 0.9324 & 0.9345 & 0.9256 & 0.9345 & 0.9401 \\
\hline & 8 & 0.9667 & 0.9612 & 0.9642 & 0.9586 & 0.9652 & 0.9710 \\
\hline & 10 & 0.9862 & 0.9792 & 0.9812 & 0.9702 & 0.9801 & 0.9875 \\
\hline & 12 & 0.9907 & 0.9855 & 0.9874 & 0.9842 & 0.9892 & 0.9924 \\
\hline \multirow[t]{4}{*}{ Image 6} & 5 & 0.8194 & 0.8176 & 0.8188 & 0.8102 & 0.8186 & 0.8213 \\
\hline & 8 & 0.9079 & 0.8844 & 0.8957 & 0.8712 & 0.8989 & 0.9086 \\
\hline & 10 & 0.9418 & 0.9158 & 0.9356 & 0.9086 & 0.9345 & 0.9486 \\
\hline & 12 & 0.9572 & 0.9449 & 0.9489 & 0.9386 & 0.9496 & 0.9601 \\
\hline
\end{tabular}




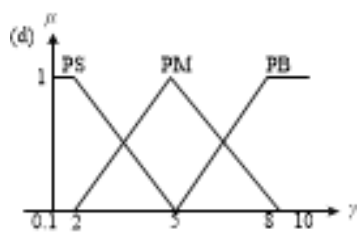

FA encounter the premature convergence whereas only FaFA overcomes the problem, and obtains the best objective function values. It also demonstrates that the success of the fuzzy adaptive strategy is effective. In conclusion, the proposed algorithm achieves significant improvement based on the standard FA, and the other five algorithms seem a little difference in convergence performance.

\subsection{Statistical Results Analysis}

A Wilcoxon rank sum test is performed to verify the experimental results, which has been conducted with 5\% significance level (Tan et al. 2021). The null hypothesis is expressed as: there is no significant difference between the FaFA algorithm and other five heuristic algorithms. Nevertheless, the alternative hypothesis deems a significant difference among them. Based on the Wilcoxon rank sum test, the $p$ value is used to judge the null hypothesis whether to accept the null hypothesis or not. if the $p$ value is greater than 0.05 , the null hypothesis will be rejected, it implies there is no significant difference between all algorithms. On the contrary, if the $p$ value is less than 0.05 , the alternative hypothesis will be accepted. Table 9 shows the $p$ value results, which is executed on the objective function value, PSNR and FSIM. It can be summarized from Table 9 that the FaFA is significantly better than FA in terms of PSNR and FSIM. However, there is no significantly difference between FaFA and SSA, ODFA, BWO, NaFA for all metrics. Analyzing the whole results, the FaFA has a remarkable improvement, it is feasible and effective for multilevel thresholding color image segmentation.

\section{CONCLUSIONS AND FUTURE WORK}

The traditional exhaustive search method for solving the optimal multilevel thresholding of satellite remote sensing images is time consuming. To address this problem, a fuzzy adaptive firefly algorithm is proposed to search the multilevel thresholding for color satellite image. In order to enhance the performance of the original FA, a fuzzy logic controller is designed. First, the ranges of the parameters are deduced. Then analyzing the change processes of the parameters. At last, a fuzzy rule tables is established and fuzzy controller is realized. The experiments results indicate the improvement of FaFA is successful. In the experiments, the proposed algorithm shows obvious improvement when compared with the other five well-known optimization algorithms for multilevel thresholding in terms of the objective function value, standard deviation, PSNR, FSIM and convergence performance. Besides,

Table 9. p-value test results

\begin{tabular}{|l|l|l|l|l|l|}
\hline \multirow{2}{*}{ Metrics } & \multicolumn{5}{|c|}{ Versus FaFA $p$ Value } \\
\cline { 2 - 6 } & \multicolumn{1}{|c|}{ NaFA } & \multicolumn{1}{|c|}{ SSA } & \multicolumn{1}{c|}{ ODFA } & \multicolumn{1}{c|}{ FA } & BWO \\
\hline OF & 0.7807 & 0.5847 & 0.6575 & 0.5706 & 0.7028 \\
\hline PSNR & 0.7182 & 0.0889 & 0.4393 & $\mathbf{0 . 0 4 0 2}$ & 0.6575 \\
\hline FSIM & 0.5990 & 0.1147 & 0.2882 & $\mathbf{0 . 0 3 2 8}$ & 0.3223 \\
\hline
\end{tabular}


the Wilcoxon's rank sum test further proves the validity of the proposed algorithm. However, the advantages of the proposed algorithm is not very obvious. Future works of this study will focus on studying the performance of the proposed algorithm for higher numbers of thresholds, and adopting different entropy functions. 


\section{ACKNOWLEDGMENT}

This work is supported by the Natural Science Foundation of Guangdong Province of China with the Grant No.2020A1515010784.

\section{REFERENCES}

Aziz, M. A., Ewees, A. A., \& Hanien, A. E. (2017). Whale Optimization Algorithm and Moth-Flame Optimization for multilevel thresholding image segmentation. Expert Systems with Applications, 83, 242-256. doi:10.1016/j.eswa.2017.04.023

Bao, X., Jia, H., \& Lang, C. (2019). A Novel Hybrid Harris Hawks Optimization for Color Image Multilevel Thresholding Segmentation. IEEE Access: Practical Innovations, Open Solutions, 7, 76529-76546. doi:10.1109/ ACCESS.2019.2921545

Baykasoglu, A., \& Ozsoydan, F. B. (2015). Adaptive firefly algorithm with chaos for mechanical design optimization problems. Applied Soft Computing, 36, 152-164. doi:10.1016/j.asoc.2015.06.056

Beevi, K. S., Nair, M. S., \& Bindu, G. R. (2016). Automatic segmentation of cell nuclei using Krill Herd optimization based multi-thresholding and Localized Active Contour Model. Biocybernetics and Biomedical Engineering, 36(4), 584-596. doi:10.1016/j.bbe.2016.06.005

Bhandari, A. K. (2018). A novel beta differential evolution algorithm-based fast multilevel thresholding for color image segmentation. Neural Computing \& Applications, 1-31. doi:10.1007/s00521-018-3771-z

Dhal, K. G., Das, A., \& Ray, S. (2019). Nature-Inspired Optimization Algorithms and Their Application in Multi-Thresholding Image Segmentation. Archives of Computational Methods in Engineering, 1-34. doi:10.1007/ s11831-019-09334-y

Elaziz, M. A., \& Lu, S. (2019). Many-objectives multilevel thresholding image segmentation using Knee Evolutionary Algorithm. Expert Systems with Applications, 125, 305-316. doi:10.1016/j.eswa.2019.01.075

Fister, I. Jr, Perc, M., Kamal, S. M., \& Fister, I. (2015). A review of chaos-based firefly algorithms. Applied Mathematics and Computation, 252, 155-165. doi:10.1016/j.amc.2014.12.006

Horng, M. H. (2011). Multilevel thresholding selection based on the artificial bee colony algorithm for image segmentation. Expert Systems with Applications, 38(11), 13785-13791. doi:10.1016/j.eswa.2011.04.180

Houssein, E. H., Helmy, B. E., Oliva, D., Elngar, A. A., \& Shaban, H. (2020). A novel Black Widow Optimization algorithm for multilevel thresholding image segmentation. Expert Systems with Applications, 167, 114159. doi:10.1016/j.eswa.2020.114159

Ibrahim, I. A., \& Khatib, T. (2017). A novel hybrid model for hourly global solar radiation prediction using random forests technique and firefly algorithm. Energy Conversion and Management, 138, 413-425. doi:10.1016/j. enconman.2017.02.006

Jia, H., Lang, C., Oliva, D., Song, W., \& Peng, X. (2019). Hybrid Grasshopper Optimization Algorithm and Differential Evolution for Multilevel Satellite Image Segmentation. Remote Sensing, 11(9), 1134. doi:10.3390/ rs11091134

Khairuzzaman, A. K., \& Chaudhury, S. (2017a). Moth-Flame Optimization Algorithm Based Multilevel Thresholding for Image Segmentation. International Journal of Applied Metaheuristic Computing, 8(4), 58-83. doi:10.4018/IJAMC.2017100104

Khairuzzaman, A. K., \& Chaudhury, S. (2017b). Multilevel thresholding using grey wolf optimizer for image segmentation. Expert Systems with Applications, 86, 64-76. doi:10.1016/j.eswa.2017.04.029

Khorram, B., \& Yazdi, M. (2019). A New Optimized Thresholding Method Using Ant Colony Algorithm for MR Brain Image Segmentation. Journal of Digital Imaging, 32(1), 162-174. doi:10.1007/s10278-018-0111-x PMID:30091112 
Li, K., Chen, Y., Li, W., He, J., \& Xue, Y. (2018). Improved gene expression programming to solve the inverse problem for ordinary differential equations. Swarm and Evolutionary Computation, 38, 231-239. doi:10.1016/j. swevo.2017.07.005

Li, K., Liang, Z., Yang, S., Chen, Z., Wang, H., \& Lin, Z. (2019). Performance Analyses of Differential Evolution Algorithm Based on Dynamic Fitness Landscape. International Journal of Cognitive Informatics and Natural Intelligence, 13(1), 36-61. doi:10.4018/IJCINI.2019010104

Li, K., Wang, H., \& Li, S. (2019). A mobile node localization algorithm based on an overlapping self-adjustment mechanism. Information Sciences, 481, 635-649. doi:10.1016/j.ins.2018.12.006

Manikandan, S., Ramar, K., \& Iruthayarajan, M. W. (2014). Multilevel thresholding for segmentation of medical brain images using real coded genetic algorithm. Measurement, 47, 558-568. doi:10.1016/j. measurement.2013.09.031

Naidu, M. S., Kumar, P. R., \& Chiranjeevi, K. (2017). Shannon and Fuzzy entropy based evolutionary image thresholding for image segmentation. Alexandria Engineering Journal, 57(3), 1643-1655.

Pare, S., Bhandari, A. K., \& Kumar, A. (2017). A new technique for multilevel color image thresholding based on modified fuzzy entropy and Lévy flight firefly algorithm. Computers \& Electrical Engineering, 476-495.

Pare, S., Kumar, A., Singh, G. K., \& Bajaj, V. (2020). Image Segmentation Using Multilevel Thresholding: A Research Review. Iranian Journal of Science and Technology. Transaction of Electrical Engineering, 44(1), 1-29. doi:10.1007/s40998-019-00251-1

Raja, N. S., Manic, K. S., \& Rajinikanth, V. (2013). Firefly Algorithm with Various Randomization Parameters: An Analysis. Swarm Evolutionary and Memetic Computing, 110-121.

Rajinikanth, V., \& Couceiro, M. S. (2015). RGB Histogram Based Color Image Segmentation Using Firefly Algorithm. Procedia Computer Science, 46, 1449-1457. doi:10.1016/j.procs.2015.02.064

Song, S., Jia, H., \& Ma, J. (2019). A chaotic electromagnetic field optimization algorithm based on fuzzy entropy for multilevel thresholding color image segmentation. Entropy (Basel, Switzerland), 21(4), 398. doi:10.3390/ e21040398 PMID:33267113

Suresh, S., \& Lal, S. (2017). Multilevel thresholding based on Chaotic Darwinian Particle Swarm Optimization for segmentation of satellite images. Applied Soft Computing, 55, 503-522. doi:10.1016/j.asoc.2017.02.005

Tan, Z., Li, K., \& Wang, Y. (2021). Differential Evolution With Adaptive Mutation Strategy Based on Fitness Landscape Analysis. Information Sciences, 549, 142-163. doi:10.1016/j.ins.2020.11.023

Tang, K., Xiao, X., Wu, J., Yang, J., \& Luo, L. (2017). An improved multilevel thresholding approach based modified bacterial foraging optimization. Applied Intelligence, 46(1), 214-226. doi:10.1007/s10489-016-0832-9

Upadhyay, P., \& Chhabra, J. K. (2019). Image Segmentation Using Electromagnetic Field Optimization (EFO) in E-Commerce Applications. International Journal of Information System Modeling and Design, 10(3), 76-91. doi:10.4018/IJISMD.2019070105

Verma, O. P., Aggarwal, D., \& Patodi, T. (2016). Opposition and dimensional based modified firefly algorithm. Expert Systems with Applications, 44, 168-176. doi:10.1016/j.eswa.2015.08.054

Wang, G., Li, W., Zuluaga, M. A., Pratt, R., Patel, P. A., Aertsen, M., Doel, T., David, A. L., Deprest, J., Ourselin, S., \& Vercauteren, T. (2018). Interactive Medical Image Segmentation Using Deep Learning With Image-Specific Fine Tuning. IEEE Transactions on Medical Imaging, 37(7), 1562-1573. doi:10.1109/TMI.2018.2791721 PMID:29969407

Wang, H., Wang, W., Zhou, X., Sun, H., Zhao, J., Yu, X., \& Cui, Z. (2017). Firefly algorithm with neighborhood attraction. Information Sciences, 382-383, 374-387. doi:10.1016/j.ins.2016.12.024

Wang, R., Zhou, Y., Zhao, C., \& Wu, H. (2015). A hybrid flower pollination algorithm based modified randomized location for multi-threshold medical image segmentation. Bio-Medical Materials and Engineering, 26(s1), S1345-S1351. doi:10.3233/BME-151432 PMID:26405895 
Wang, S., Jia, H., \& Peng, X. (2020). Modified salp swarm algorithm based multilevel thresholding for color image segmentation. Mathematical Biosciences and Engineering, 17(1), 700-724. doi:10.3934/mbe.2020036 PMID:31731372

Yang, X. (2009). Firefly algorithms for multimodal optimization. International Conference on Stochastic Algorithms Foundations and Applications, 169-178.

Yi Wang is currently a full lecturer from Guangdong university of science and technology. She received master's degree from the Guangxi normal university, also received the bachelor's degree from Huahua college. Her research interests include evolutionary optimization, image processing. She has published over 10 research papers.

Kangshun Li is currently a Full Professor from the South China Agricultural University, Guangzhou, China. He received his BSc in Computational Mathematics from the Nanchang University, Nanchang, China, and PhD in Computer Software and Theory from the Wuhan University, Wuhan, China, in 1983 and 2006, respectively. His current research interests are intelligent computation, image identification, data mining, soft engineering, embedded system, evolvable hardware, evolutionary modelling, parallel computation, and neural network. 